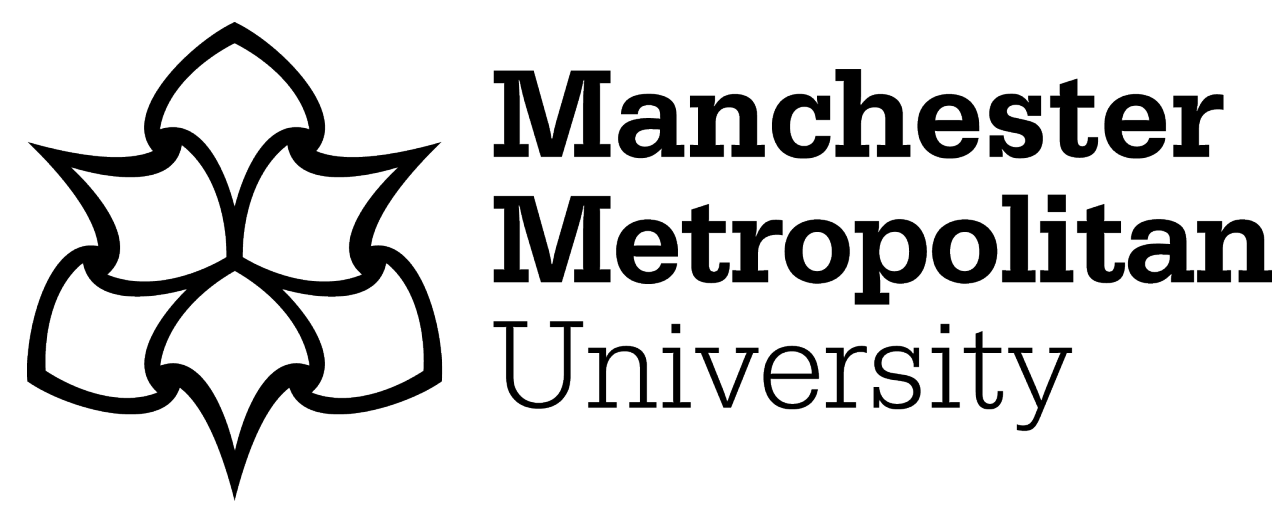

Jamshidi, Rashid ORCID logoORCID: https://orcid.org/0000-0001-84078005 and Mazzei, Luca (2018) CFD Modeling of Fluidized Beds. Reference Module in Chemistry, Molecular Sciences and Chemical Engineering.

Downloaded from: https://e-space.mmu.ac.uk/625164/ Version: Accepted Version Publisher: Elsevier

DOI: https://doi.org/10.1016/B978-0-12-409547-2.13698-4

Usage rights: Creative Commons: Attribution-Noncommercial-No Derivative Works 4.0

Please cite the published version 


\title{
CFD Modeling of Fluidized Beds
}

\author{
Rashid Jamshidi, Luca Mazzei* \\ Department of Chemical Engineering, University College London, Torrington Place, London WC1E 7JE, UK
}

\section{Contents}

I Introduction $\quad 2$

II A brief overview of fluidized bed modeling $\quad 3$

III Averaged equations of motion for fluid-particle systems 4

$\begin{array}{ll}\text { IV The closure problem } & 10\end{array}$

$\begin{array}{lll}\text { V Population balance modeling } & 17\end{array}$

$\begin{array}{ll}\text { VI Conclusions } & 19\end{array}$

$\begin{array}{ll}\text { Bibliography } & 19\end{array}$

\begin{abstract}
We review the mathematical modeling of fluidized suspensions with focus on the Eulerian (or multifluid) approach. After a brief survey of different modeling approaches adopted for multiphase flows, we discuss the Eulerian equations of motion for fluidized suspensions of a finite number of monodisperse particle classes, obtained by volume averaging. We present the problem of closure for the stress tensors and the interaction forces between the phases and report some of the constitutive relations used for them in the literature. Finally, we explain briefly the population balance modeling approach, which allows handling suspensions of particles continuously distributed over any of their properties of interest.
\end{abstract}

Keywords: Closure problem, Computational fluid dynamics, Fluidization, Fluidized bed, Fluid-particle interaction force, Multifluid modeling, Multiphase flows, Volume averaging.

*Email address: 1.mazzei@ucl.ac.uk. Tel: +44 (0)20 7679 4328, Fax: +44 (0)20 73832348. 


\section{Introduction}

Fluidized beds are granular systems of solid particles subjected to a vertical upward flow of fluid. They have been used in several industrial processes for almost a century. The reason for their popularity is simple: the fluid flow, above a threshold value, suspends the particles, because the interaction force between these and the fluid overcomes the particle weight; in this "fluidized" condition, the bed acts as a highly efficient mixer, provides high mass and heat transfer rates (thereby rendering the temperature of the system nearly uniform) and is able to operate continuously without expensive repairing costs. Moreover, the large surface-to-volume ratio for the solid phase considerably enhances the rate of chemical reactions. These benefits have rendered fluidized beds the technology of choice in a large number of key industrial processes, such as fluid catalytic cracking (FCC), food processing, coal combustion and biomass gasification.

The characteristic length of industrial fluidized beds usually varies between few to several meters (Ismail et al., 2016; Massoudi et al., 2017; Zhao et al., 2017); nevertheless, the fluid dynamics of fluidized powders are affected by processes taking place at many length scales, the smallest being shorter than the particle size. This complexity renders fluidized beds difficult to model; for this reason, historically, their design was based on a practical approach that relied on pilot plans and experimental observations (refer, for instance, to Leva, 1959). It should be mentioned, however, that in the first processes in which fluidized beds were employed, the required plant performance was either not important (such as in FCC plants) or easily achievable (such as in roasting and drying). Later, owing to the low plant efficiency in more demanding applications, researchers endeavored to find more reliable methods to predict the dynamics of fluidized suspensions. The motivation was also that the empirical correlations obtained from pilot plants suffered from lack of general validity, and therefore in most cases they could not assist in the design of beds with new geometries, configurations of heat exchanger tubes or repartitions of the feed over several entry points. To overcome these problems, a few of which we reported as examples, one should understand the theory underlying the fluid dynamics of fluidized suspensions. Pilot plants are not a convenient alternative, since they are expensive, time-consuming and not always helpful for scale up calculations.

Theoretical models for fluidized powders (and more in general multiphase flows) started to appear in the 1960s, when scientists began to use the conservation laws of mass, momentum and energy for these systems. The influential textbook Transport Phenomena by Bird et al. (1960), one of the earliest in this area, prompted the first attempts to develop fluid dynamic models based on transport equations. Anderson \& Jackson (1967) were one of the first to use this approach to model fluidized beds. Starting from the continuity and dynamical equations for single-phase, incompressible fluids and from the Newtonian equations for rigid-body motion, they derived averaged balance equations for the fluid and particle phases by applying a formal mathematical process of volume averaging. Subsequently, other researchers, such as Whitaker (1969), Drew (1971) and Drew \& Segel (1971), adopted the same approach to better understand the complex behavior of multiphase systems. Of course, at that time solving the governing equations was impossible, and so theoretical modeling was not viewed as a viable way to design real industrial units. It took almost two decades for the researchers working in this field to realize that the mathematical theory of multiphase flows could be a useful design tool, since faster computer processors and advanced numerical methods to integrate partial differential equations had in the meantime become available, making the models numerically solvable.

The late 1970s and early 1980s were the years in which the results of the first numerical simulations of granular flows appeared in the literature (Pritchett et al., 1978; Gidaspow \& Ettehadieh, 1983; Gidaspow et al., 1986). These pioneering studies showed that computational fluid dynamics (CFD) is a powerful tool for understanding the physics of multiphase flows, and this increased quite rapidly the interest of the research community in this numerical approach. It is worth mentioning that other rigorous formulations of multiphase equations of motion were also developed at the same time, see, for instance, Buyevich (1971), Hinch (1977), Nigmatulin (1979), Drew (1983) and Jenkins \& Savage (1983). After almost three decades, today, in the field of fluidization, CFD has become a nearly irreplaceable tool to predict many of the macroscopic phenomena encountered in particulate systems, having significantly assisted to understand fluid-solid interactions.

Recently, this macroscopic tool has found other counterparts, such as the Lattice Boltzmann Method and the Discrete Element Method. These are able to tackle the same problem (i.e., the fluid dynamic description 
of fluidized suspensions) at smaller scales (the mesoscale and microscale, respectively). In the next section, we briefly overview some of these modeling approaches.

\section{A brief overview of fluidized bed modeling}

Computational methods are able to offer useful information about the physical behavior of multiphase flows. These flows, as said, feature a multitude of length and time scales. While in theory a model could capture all of them, giving a fluid dynamic description of the system down to the smallest detail, doing so is impractical, extremely demanding computationally and often unnecessary. Thus, several modeling approaches have been developed that, by separating the scales, reduce the complexity of the multiscale problem. For the particulate phase, two main modeling strategies are possible: treating the phase as a continuum or treating each particle individually. The former approach results in transport equations similar to those that govern the dynamics of single-phase media and is called Eulerian modeling approach, while the latter preserves the discrete nature of the particulate phase and is referred to as Lagrangian modeling approach. As we discuss below, each of these approaches presents advantages and disadvantages.

The most fundamental level of modeling, which in principle could capture all the length and time scales characterizing a fluid-particle system, is referred to as Eulerian-Lagrangian and considers the fluid phase as a continuum (Eulerian) and the solid phase as a population of particles (Lagrangian). In models of this kind, the continuity and Navier-Stokes equations are to be satisfied at every point occupied by the interstitial fluid, and the Newtonian equations for translation and rotation are solved for each particle. This approach models the fluid phase around the particles at a length scale far smaller than the particle size. One therefore obtains the distributions of fluid pressure and velocity over the surface of the particles, and hence can determine the interaction force between the fluid and the particles (see, for instance, Pan et al., 2002 and Feng et al., 2016). The considerable advantage of this "fully resolved" approach is that there is no closure problem regarding the interaction force between the phases. Moreover, since the particles are considered individually, the equations describing their motion feature no granular stress; therefore, the closure problem for this term, which arises in macroscopic models and is extremely complex, is absent. However, in spite of such benefits, this modeling strategy is excessively demanding computationally and, at present, is still not viable for describing industrial fluidized beds. Furthermore, the detailed information that this approach yields is not of direct use to process engineers and requires filtering to deliver observables of interest, such as volume fractions, mean velocities, granular temperature and granular pressure.

To overcome the problem just mentioned and decrease the computational costs, it is more convenient to treat all the phases as continua. This modeling approach, called Eulerian-Eulerian or multifluid, is based on a formal mathematical procedure of averaging and is able to predict the evolution of the observables directly. The dynamic description of the system is considerably reduced, but the information that usually is of interest in engineering applications can be captured with sufficient detail. The main drawback of this approach is that the averaged transport equations are unclosed, because the averaging process generates terms which are still related to the dynamics of the system at length scales smaller than that characterizing the mean fields. These terms are the stress tensors of the phases and the interaction force between the phases. Constitutive equations for them cannot be obtained analytically, with the exception of relatively simple limiting cases; thus, in most flows, empirical or semi-empirical relations must be adopted. In the following sections of this article, we will discuss in more detail the derivation of the multifluid averaged equations and the closure problem, presenting some of the constitutive equations used to close Eulerian-Eulerian models.

A hybrid method between the Eulerian-Lagrangian and the Eulerian-Eulerian also exists. Called discrete particle modeling (DPM) approach or also hybrid Eulerian-Lagrangian modeling approach, this modeling strategy uses averaged transport equations for the interstitial fluid phase and rigid-body Newtonian equations for the particles. The closure problem related to the solid-phase stress is therefore eliminated; however, those related to the (average) fluid-phase stress and to the interaction force between the phases remain. This is what distinguishes these hybrid models from the Eulerian-Lagrangian models previously described (in which no closure problem arises). In the latter, for instance, the overall force exerted by the fluid on each particle can 
be determined by integrating over the particle surface the local traction related to the fluid velocity gradients, which the model rigorously captures. Therefore, the fluid-particle interaction force is closed. Conversely, in the hybrid models the force is related to the slip velocity between the average fluid velocity and the velocity of the particle center of mass and is obtained by means of empirical relations. The hybrid modeling approach is significantly less demanding computationally than the Eulerian-Lagrangian and has been used extensively to simulate fluidized powders (Tsuji et al., 1993; Hoomans et al., 1996; Xu \& Yu, 1997; Ouyang \& Li, 1999; Kafui et al., 2002; Lu et al., 2005; Zhu et al., 2008; Di Renzo et al., 2011; He et al., 2012; Wang et al., 2013; Deen et al., 2014; Schneiderbauer et al., 2015, 2016).

Among the modeling approaches discussed, the Eulerian-Eulerian is the most suitable for industrial scale fluidized beds, insofar as it is the least demanding computationally and provides directly - that is, without the need of filtering - information about measurable variables, such as void fraction and granular pressure, that are of interest to end-users. Owing to the limitations that most computer processors still present, Lagrangian models are unlikely to replace Eulerian ones in the near future. Lagrangian models, however, are extremely valuable in research; yielding detailed information about the dynamics of multiphase flows at the mesoscopic and microscopic length scales, they can significantly help to derive and improve continuous models through the derivation of accurate closure relations. To do so, nevertheless, one needs to link shorter-scale models to larger-scale ones, extracting information from the former to overcome closure problems in the latter. This is far from simple and still an open challenge.

\section{Averaged equations of motion for fluid-particle systems}

In this section, we describe the averaged balance equations for mass and linear momentum for fluidized beds. We assume that the system is isothermal and that the flow is laminar; therefore, we do not consider equations for energy and turbulence. The general form of the transport equations is valid for other types of multiphase systems, not just fluidized beds; what renders the equations specific to a particular system (e.g., fluid-solid or fluid-fluid) is the constitutive relations adopted to close the equations.

Three averaging methods are commonly adopted to derive the averaged balance equations: ensemble, or statistical, averaging (e.g., Sangani \& Didwania, 1993; Zhang \& Prosperetti, 1994; Marchioro et al., 1999), time averaging, which includes Reynolds averaging and density-weighted time (Favre) averaging (e.g., Ishii \& Mishima, 1984; Gidaspow, 1994; Ishii \& Takashi, 2010) and volume averaging (e.g. Anderson \& Jackson, 1967; Nigmatulin, 1979; Hwang \& Shen, 1989; Jackson, 1997). Each of these methods has advantages and disadvantages; as a discussion of this aspect exceeds the scope of this article, we refer the interested reader to Jackson (2000). It is worth mentioning that different averaging schemes might lead to different, but similar, evolution equations, which present common features. For example, similar equations obtained from volume averaging can be obtained with ensemble and time averaging (refer, for instance, to Gidaspow, 1994, Zhang \& Prosperetti, 1994, Drew \& Passman, 1998 and Brilliantov \& Poschel, 2004). If the average values given by different techniques are equal, the system is said to be ergodic, but not all systems enjoy this feature. Further information about this is found in Jackson (2000).

In this review, we focus on the volume averaging method. In this method, observables are computed over spatial domains that are large enough to contain a statistically-significant number of particles, but which are small compared with the length scale of variation of the observables. The mathematical tool that one can use to obtain these averages is called "weighting function". This is a monotonically decreasing continuous scalar function of the radial distance $r$ from the point $x$ in which the averaged value is to be calculated. It is positive for any $r$, has continuous derivatives of any order and is normalized in such a way that:

$$
\int_{\Omega_{x}} \psi(|\boldsymbol{x}-\boldsymbol{z}|) d \boldsymbol{z}=4 \pi \int_{0}^{\infty} \psi(r) r^{2} d r=1
$$

where $\Omega_{x}$ is the spatial domain occupied by the system of interest (assumed to extend to infinity), $\psi(|\boldsymbol{x}-\boldsymbol{z}|)$ is the weighting function, $\boldsymbol{x}$ and $\boldsymbol{z}$ are generic spatial points, and $r$ is the distance between such points. This function identifies an "averaging volume" $V_{x}$ outside which the values of the variable being averaged do not 
affect significantly that of the mean variable. This volume $V_{x}$ can be taken to be that of a sphere with radius equal to the length scale characterizing the spatial decay of the weighting function (i.e., the length over which the weighting function decreases significantly). Using this function, one can define fluid-phase and particlephase volume averages (Anderson \& Jackson, 1967; Jackson, 1997).

The volume fraction of fluid and the fluid-phase volume average of a point variable $\boldsymbol{\zeta}(\boldsymbol{x}, t)$ calculated in $\boldsymbol{x}$ at time $t$ are defined as follows:

$$
\varepsilon(\boldsymbol{x}, t) \equiv \int_{\Lambda_{e}} \psi(|\boldsymbol{x}-\boldsymbol{z}|) d \boldsymbol{z} \quad ; \quad\langle\boldsymbol{\zeta}\rangle_{e}(\boldsymbol{x}, t) \equiv \frac{1}{\varepsilon(\boldsymbol{x}, t)} \int_{\Lambda_{e}} \boldsymbol{\zeta}(\boldsymbol{z}, t) \psi(|\boldsymbol{x}-\boldsymbol{z}|) d \boldsymbol{z}
$$

where $\Lambda_{e}$ is the domain occupied by the fluid phase at time $t$.

The number density of particles of class $\mathscr{S}_{r}$ and the particle-phase volume average of a particle property $\boldsymbol{\zeta}_{r}(t)$ calculated in $\boldsymbol{x}$ at time $t$ are defined as follows:

$$
n_{r}(\boldsymbol{x}, t) \equiv \sum_{\mathscr{S}_{r}} \psi\left(\left|\boldsymbol{x}-\boldsymbol{z}_{r}(t)\right|\right) \quad ; \quad\langle\boldsymbol{\zeta}\rangle_{p}^{r}(\boldsymbol{x}, t) \equiv \frac{1}{n_{r}(\boldsymbol{x}, t)} \sum_{\mathscr{S}_{r}}\left[\boldsymbol{\zeta}_{r}(t) \psi\left(\left|\boldsymbol{x}-\boldsymbol{z}_{r}(t)\right|\right)\right]
$$

where $\boldsymbol{z}_{r}(t)$ is the position occupied at time $t$ by the center of mass of a generic particle of solid phase $\mathscr{S}_{r}$. The subscript $p$ in $\langle\zeta\rangle_{p}^{r}$ indicates that this is a particle-phase volume average, while the superscript $r$ indicates that the average refers to solid phase $\mathscr{S}_{r}$. The volume fraction $\phi_{r}(\boldsymbol{x}, t)$ of this particle class can be calculated (with good approximation; see Jackson, 1997) with the following relation:

$$
\phi_{r}(\boldsymbol{x}, t) \approx n_{r}(\boldsymbol{x}, t) V_{r}
$$

where $V_{r}$ is the volume of a particle of solid phase $\mathscr{S}_{r}$.

Extending the work of Jackson (2000) and Owoyemi et al. (2007), we now derive the averaged equations of motion for a fluid-particle system of $\nu$ solid phases; below, we use the indices $r$ and $s$ to specify the type of phase and the indices $e$ and $p$ to specify the type of volume average.

\section{A Fluid phase}

As said before, the volume-averaged equations of motion include only the continuity and linear momentum equations, since the system is assumed to be isothermal. The starting point is writing the continuity equation for the fluid phase at the microscopic scale, which is written in terms of fluid "point" velocity. This equation for an incompressible fluid reads:

$$
\partial_{\boldsymbol{x}} \cdot \boldsymbol{u}=0
$$

where $\boldsymbol{u}(\boldsymbol{x}, t)$ denotes the fluid point velocity vector. Here, we restrict the treatment to incompressible fluids for simplicity (although generalizing the analysis is not particularly complex); in many fluidized beds, even if the fluidizing medium is a gas, this approximation is acceptable. If we apply the volume averaging scheme to Eq. III.5, we obtain:

$$
\varepsilon\left\langle\partial_{\boldsymbol{x}} \cdot \boldsymbol{u}\right\rangle_{e}=0
$$

Even if correct, this equation is not practical. What we are after is an equation governing the evolution of the volume-averaged fluid velocity $\langle\boldsymbol{u}\rangle_{e}$. Clearly, Eq. III.6 is not such an equation, because, instead of featuring the divergence of the volume-averaged velocity field, it features the volume average of the divergence of the point velocity field. To obtain an evolution equation in terms of $\langle\boldsymbol{u}\rangle_{e}$, we manipulate Eq. III.6 employing the following mathematical relations:

$$
\varepsilon\left\langle\partial_{\boldsymbol{x}} \cdot \boldsymbol{\zeta}\right\rangle_{e}=\partial_{\boldsymbol{x}} \cdot\left(\varepsilon\langle\boldsymbol{\zeta}\rangle_{e}\right)-\sum_{r=1}^{\nu} \sum_{\mathscr{S}_{r}} \int_{\partial \Lambda_{r}} \boldsymbol{k}(\boldsymbol{z}, t) \cdot \boldsymbol{\zeta}(\boldsymbol{z}, t) \psi(|\boldsymbol{x}-\boldsymbol{z}|) d \boldsymbol{s}_{z}
$$




$$
\varepsilon\left\langle\partial_{t} \boldsymbol{\zeta}\right\rangle_{e}=\partial_{t}\left(\varepsilon\langle\boldsymbol{\zeta}\rangle_{e}\right)+\sum_{r=1}^{\nu} \sum_{\mathscr{S}_{r}} \int_{\partial \Lambda_{r}} \boldsymbol{k}(\boldsymbol{z}, t) \cdot \boldsymbol{u}(\boldsymbol{z}, t) \boldsymbol{\zeta}(\boldsymbol{z}, t) \psi(|\boldsymbol{x}-\boldsymbol{z}|) d \boldsymbol{s}_{z}
$$

where $\boldsymbol{\zeta}(\boldsymbol{x}, t)$ is an arbitrary vector or tensor point variable (in Eq. III.8 it can also be a scalar variable), $\Lambda_{r}$ is the region of Euclidean space occupied by a generic particle of phase $\mathscr{S}_{r}$ at time $t$ and $\boldsymbol{k}(\boldsymbol{x}, t)$ is the outward unit normal to the surface $\partial \Lambda_{r}$ bounding $\Lambda_{r}$. If we replace $\boldsymbol{\zeta}$ with $\boldsymbol{u}$ in Eq. III.7 and $\boldsymbol{\zeta}$ with unity in Eq. III.8 and add the two equations, we obtain:

$$
\partial_{t} \varepsilon+\partial_{\boldsymbol{x}} \cdot\left(\varepsilon\langle\boldsymbol{u}\rangle_{e}\right)=0
$$

This is the continuity equation which we sought. This is similar to the continuity equation for a single-phase compressible fluid with the fluid density replaced by the fluid volume fraction.

The volume-averaged linear momentum balance equation for the fluid can be obtained similarly; we first write the microscopic balance equation, which reads:

$$
\rho_{e}\left[\partial_{t} \boldsymbol{u}+\partial_{\boldsymbol{x}} \cdot(\boldsymbol{u} \boldsymbol{u})\right]=\partial_{\boldsymbol{x}} \cdot \boldsymbol{T}+\rho_{e} \boldsymbol{g}
$$

in which $\rho_{e}$ is the (constant) fluid density, $\boldsymbol{T}(\boldsymbol{x}, t)$ is the point fluid stress tensor and $\boldsymbol{g}$ is the gravity vector. We then multiply both sides of this equation by the weighting function and integrate the result with respect to the variable $z$ over the region of physical space occupied by the fluid. This procedure leads again to a form of the transport equation featuring volume averages of differential operators acting on point variables. To obtain the equation in terms of differential operators acting on mean fields, we use again Eqs. III.7 and III.8, setting $\zeta \equiv \boldsymbol{u} u$ in Eq. III.7 and $\zeta \equiv \boldsymbol{u}$ in Eq. III.8, to treat the left-hand side of Eq. III.10, and $\zeta \equiv \boldsymbol{T}$ in Eq. III.7 to treat the right-hand side. The result is:

$$
\begin{gathered}
\rho_{e}\left[\partial_{t}\left(\varepsilon\langle\boldsymbol{u}\rangle_{e}\right)+\partial_{\boldsymbol{x}} \cdot\left(\varepsilon\langle\boldsymbol{u} \boldsymbol{u}\rangle_{e}\right)\right] \\
=\partial_{\boldsymbol{x}} \cdot\left(\varepsilon\langle\boldsymbol{T}\rangle_{e}\right)+\varepsilon \rho_{e} \boldsymbol{g}-\sum_{r=1}^{\nu} \sum_{\mathscr{S}_{r}} \int_{\partial \Lambda_{r}} \boldsymbol{k}(\boldsymbol{z}, t) \cdot \boldsymbol{T}(\boldsymbol{z}, t) \psi(|\boldsymbol{x}-\boldsymbol{z}|) d \boldsymbol{s}_{z}
\end{gathered}
$$

As we can see, in the averaged equation an extra term appears on the right-hand side. This is the sum over all particle classes of the average resultant traction forces exerted by the fluid on the particles of each class. The exterior summation is over all particle classes and the term:

$$
\sum_{\mathscr{S}_{r}} \int_{\partial \Lambda_{r}} \boldsymbol{k}(\boldsymbol{z}, t) \cdot \boldsymbol{T}(\boldsymbol{z}, t) \psi(|\boldsymbol{x}-\boldsymbol{z}|) d \boldsymbol{s}_{z}
$$

is the average resultant force exerted by the fluid on the particles of class $r$. To determine this force, one first calculates the mean resultant force exerted by the fluid on a generic particle belonging to the particle class $r$ (this is given by the integral in the equation above) and then sums this force over all the particles of the class considered. To find the mean resultant force over a single particle, one first weights the differential traction forces acting on each differential region $d s_{z}$ of the particle surface using the values of the weighting function corresponding to each region and then sums the (infinite number of) contributions. The term in Eq. III.12 is therefore the interaction force between the fluid phase and the $r$ th solid phase, which couples the dynamical equation of the fluid to that of each particle class.

We can manipulate the interaction force in Eq. III.12 to provide more insight into the linear momentum exchange between the particles and the fluid. To do so, we expand the weighting function in a Taylor series about the center $\boldsymbol{z}_{r}(t)$ of a generic particle of phase $\mathscr{S}_{r}$. This yields:

$$
\begin{gathered}
\forall \boldsymbol{z} \in \partial \Lambda_{r}: \psi(|\boldsymbol{x}-\boldsymbol{z}|)=\psi\left(\left|\boldsymbol{x}-\boldsymbol{z}_{r}\right|\right) \\
-\boldsymbol{k}(\boldsymbol{z}) \cdot\left[\partial_{\boldsymbol{x}} \psi\left(\left|\boldsymbol{x}-\boldsymbol{z}_{r}\right|\right)\right] r_{r}+(1 / 2) \boldsymbol{k}(\boldsymbol{z}) \boldsymbol{k}(\boldsymbol{z}) \cdot \cdot\left[\partial_{\boldsymbol{x}} \partial_{\boldsymbol{x}} \psi\left(\left|\boldsymbol{x}-\boldsymbol{z}_{r}\right|\right)\right] r_{r}^{2}-O\left(r_{r}^{3}\right)
\end{gathered}
$$


in which $r_{r}$ is the radius of the particles of phase $\mathscr{S}_{r}$. We know that the particle radius is far smaller than the radius of the averaging volume $V_{x}$ and therefore, with acceptable error, we may truncate the Taylor series at the second-order term. Thus, using Eq. III.13, we approximate the force in Eq. III.12 as:

$$
n_{r}\langle\boldsymbol{f}\rangle_{p}^{r}-\partial_{\boldsymbol{x}} \cdot\left(n_{r}\langle\boldsymbol{A}\rangle_{p}^{r}\right)+(1 / 2) \partial_{\boldsymbol{x}} \partial_{\boldsymbol{x}} \cdot \cdot\left(n_{r}\langle\boldsymbol{B}\rangle_{p}^{r}\right)
$$

in which:

$$
\begin{gathered}
n_{r}(\boldsymbol{x}, t)\langle\boldsymbol{f}\rangle_{p}^{r}(\boldsymbol{x}, t) \equiv \sum_{\mathscr{S}_{r}}\left[\psi\left(\left|\boldsymbol{x}-\boldsymbol{z}_{r}\right|\right) \int_{\partial \Lambda_{r}} \boldsymbol{k}(\boldsymbol{z}, t) \cdot \boldsymbol{T}(\boldsymbol{z}, t) d \boldsymbol{s}_{z}\right] \\
n_{r}(\boldsymbol{x}, t)\langle\boldsymbol{A}\rangle_{p}^{r}(\boldsymbol{x}, t) \equiv \sum_{\mathscr{S}_{r}}\left[\psi\left(\left|\boldsymbol{x}-\boldsymbol{z}_{r}\right|\right) r_{r} \int_{\partial \Lambda_{r}} \boldsymbol{k}(\boldsymbol{z}, t) \boldsymbol{k}(\boldsymbol{z}, t) \cdot \boldsymbol{T}(\boldsymbol{z}, t) d \boldsymbol{s}_{z}\right] \\
n_{r}(\boldsymbol{x}, t)\langle\boldsymbol{B}\rangle_{p}^{r}(\boldsymbol{x}, t) \equiv \sum_{\mathscr{S}_{r}}\left[\psi\left(\left|\boldsymbol{x}-\boldsymbol{z}_{r}\right|\right) r_{r}^{2} \int_{\partial \Lambda_{r}} \boldsymbol{k}(\boldsymbol{z}, t) \boldsymbol{k}(\boldsymbol{z}, t) \boldsymbol{k}(\boldsymbol{z}, t) \cdot \boldsymbol{T}(\boldsymbol{z}, t) d \boldsymbol{s}_{z}\right]
\end{gathered}
$$

The quantities defined above are the components of a vector, a second-order tensor and a third-order tensor, respectively. The force in Eq. III.12 is obtained by first weighting the differential traction forces exerted on the infinitesimal surface elements of the fluid-particle interface using the values of the weighting function at the locations of the elements, and then by summing such contributions. The force in Eq. III.15, conversely, is obtained by first computing the forces acting on the whole surface of each particle, then by weighting them using the values of the weighting function at the particle centers, and finally by summing such contributions. This second average interprets better the fluid-particle interaction force and fulfills the principle of action and reaction, as we will see in Section B (when we deal with the solid phases); this is why we prefer to operate in terms of this average force, and of the additional contributions present in Eq. III.14.

As we see in Eq. III.11, the convective term features the average of the dyadic product of point velocities; this quantity is not equal to the dyadic product of the average velocities. We find it convenient to decompose this term as follows:

$$
\langle\boldsymbol{u} \boldsymbol{u}\rangle_{e} \approx\langle\boldsymbol{u}\rangle_{e}\langle\boldsymbol{u}\rangle_{e}+\langle\hat{\boldsymbol{u}} \hat{\boldsymbol{u}}\rangle_{e}
$$

in which hatted variables denote the deviations of point variables from their respective average values. The above relation is not exact, being valid only when there is separation of scales between the microscopic and macroscopic descriptions of the flow. We now use Eqs. III.14 and III.18 in Eq. III.11 to obtain the final form of the linear momentum balance equation for the fluid phase; this reads:

$$
\rho_{e}\left[\partial_{t}\left(\varepsilon\langle\boldsymbol{u}\rangle_{e}\right)+\partial_{\boldsymbol{x}} \cdot\left(\varepsilon\langle\boldsymbol{u}\rangle_{e}\langle\boldsymbol{u}\rangle_{e}\right)\right]=\partial_{\boldsymbol{x}} \cdot\langle\boldsymbol{S}\rangle_{e}-\sum_{r=1}^{\nu}\left(n_{r}\langle\boldsymbol{f}\rangle_{p}^{r}\right)+\varepsilon \rho_{e} \boldsymbol{g}
$$

in which:

$$
\langle\boldsymbol{S}\rangle_{e} \equiv \varepsilon\langle\boldsymbol{T}\rangle_{e}+\sum_{r=1}^{\nu}\left[n_{r}\langle\boldsymbol{A}\rangle_{p}^{r}-(1 / 2) \partial_{\boldsymbol{x}} \cdot\left(n_{r}\langle\boldsymbol{B}\rangle_{p}^{r}\right)\right]-\varepsilon \rho_{e}\langle\hat{\boldsymbol{u}} \hat{\boldsymbol{u}}\rangle_{e}
$$

is called fluid-phase effective stress tensor. This term is unclosed and obtaining an analytical closure is very complex; this has been done only for extremely dilute systems of monodisperse Stokesian particles (see, for instance, Jackson 1997). We will discuss the closure problem in Section IV.

\section{B Solid phases}

For any arbitrary particle property $\boldsymbol{\zeta}_{r}(t)$ associated with particles of a generic solid phase $\mathscr{S}_{r}$, the following mathematical relation holds: 


$$
n_{r}\langle\dot{\boldsymbol{\zeta}}\rangle_{p}^{r}=\partial_{t}\left(n_{r}\langle\boldsymbol{\zeta}\rangle_{p}^{r}\right)+\partial_{\boldsymbol{x}} \cdot\left(n_{r}\langle\boldsymbol{v} \boldsymbol{\zeta}\rangle_{p}^{r}\right)
$$

in which the dot is a time derivative and $\langle\boldsymbol{v} \boldsymbol{\zeta}\rangle_{p}^{r}(\boldsymbol{x}, t)$ is the average of the product of $\boldsymbol{\zeta}_{r}(t)$ and of the velocity vector $\boldsymbol{v}_{r}(t)$ of the particle center. To obtain the volume-averaged continuity equation for the $r$ th solid phase, we use the equation above, setting $\boldsymbol{\zeta}_{r} \equiv 1$. This yields:

$$
\partial_{t} n_{r}+\partial_{\boldsymbol{x}} \cdot\left(n_{r}\langle\boldsymbol{v}\rangle_{p}^{r}\right)=0
$$

This equation is similar to the continuity equation of a single-phase compressible fluid, with the fluid density replaced by the particle number density.

The equation of motion for the generic solid phase $\mathscr{S}_{r}$ can be obtained from Newton's second law written for the generic particle $r$ of the phase considered. This equation reads:

$$
\rho_{r} V_{r} \dot{\boldsymbol{v}}_{r}(t)=\int_{\partial \Lambda_{r}} \boldsymbol{k}(\boldsymbol{z}, t) \cdot \boldsymbol{T}(\boldsymbol{z}, t) d \boldsymbol{s}_{z}+\sum_{s=1}^{\nu} \sum_{\mathscr{S}_{S}} \boldsymbol{f}_{r s}(t)+\rho_{r} V_{r} \boldsymbol{g}
$$

in which $\rho_{r}$ is the density of the particles of phase $\mathscr{S}_{r}, V_{r}$ is the volume of each of these particles and $\dot{\boldsymbol{v}}_{r}(t)$ is the acceleration of the particle center of mass. The surface integral on the right-hand side of the equation is the overall force exerted by the fluid on the particle. $\boldsymbol{f}_{r s}(t)$ is the force exerted on the $r$ particle by the generic $s$ particle of phase $\mathscr{S}_{s}$ when they collide. This force vanishes when $r$ and $s$ refer to the same particle, and is non-zero only if particles $r$ and $s$ are in direct contact.

To average the equation above, we multiply both sides of the equation by $\psi\left(\left|\boldsymbol{x}-\boldsymbol{z}_{r}\right|\right)$ and sum over all the particles of phase $\mathscr{S}_{r}$; after, to treat the left-hand side of the resulting equation, we use Eq. III.3, setting $\boldsymbol{\zeta}_{r} \equiv \dot{\boldsymbol{v}}_{r}$, while to treat the right-hand side we use Eq. III.21, setting $\boldsymbol{\zeta}_{r} \equiv \boldsymbol{v}_{r}$ and using the relation reported below, whose proof is left to the reader:

$$
\sum_{\mathscr{S}_{r}}\left[\psi\left(\left|\boldsymbol{x}-\boldsymbol{z}_{r}(t)\right|\right) \sum_{s=1}^{\nu} \sum_{\mathscr{S}_{s}} \boldsymbol{f}_{r s}(t)\right]=\sum_{s=1}^{\nu} \sum_{\mathscr{S}_{r}}\left[\psi\left(\left|\boldsymbol{x}-\boldsymbol{z}_{r}(t)\right|\right) \sum_{\mathscr{S}_{s}} \boldsymbol{f}_{r s}(t)\right]
$$

Doing so yields the averaged linear momentum balance equation for the solid phase $\mathscr{S}_{r}$; the equation reads:

$$
\begin{gathered}
\rho_{r} V_{r}\left[\partial_{t}\left(n_{r}\langle\boldsymbol{v}\rangle_{p}^{r}\right)+\partial_{\boldsymbol{x}} \cdot\left(n_{r}\langle\boldsymbol{v} \boldsymbol{v}\rangle_{p}^{r}\right)\right] \\
=n_{r}\langle\boldsymbol{f}\rangle_{p}^{r}+n_{r} \rho_{r} V_{r} \boldsymbol{g}+\sum_{s=1}^{\nu} \sum_{\mathscr{S}_{r}}\left[\psi\left(\left|\boldsymbol{x}-\boldsymbol{z}_{r}(t)\right|\right) \sum_{\mathscr{S}_{s}} \boldsymbol{f}_{r s}(t)\right]
\end{gathered}
$$

The first term on the right-hand side is the fluid-particle interaction force - which also features, with opposite sign, in Eq. III.19. This force fulfills the action-and-reaction principle, as it should. The final term combines the resultant forces arising from the particle-particle contacts among particles that belong to the same phase $(s=r)$ and to different phases $(s \neq r)$. These contributions are conceptually different, insofar as the former is a self-interaction term that represents the stress internal to the phase under examination, while the latter is a contact force acting between the Eulerian solid phases. To let the collisional solid stress tensor associated with phase $\mathscr{S}_{r}$ appear explicitly in Eq. III.25, we need to manipulate the equation further. We first consider the following double sum over the particles $r$ and $s$ of the $r$ th phase:

$$
\sum_{\mathscr{S}_{r}} \sum_{\mathscr{S}_{r}}\left[\psi\left(\left|\boldsymbol{x}-\boldsymbol{z}_{r s}(t)\right|\right) \boldsymbol{f}_{r s}(t)\right]
$$

where $\boldsymbol{z}_{r s}(t)$ is the position vector of the point of mutual contact between the rigid particles $r$ and $s$. Because $\boldsymbol{z}_{r s}=\boldsymbol{z}_{s r}$, this double sum is zero. Also, for the action-and-reaction principle, it is $\boldsymbol{f}_{r s}=-\boldsymbol{f}_{s r}$. Now, if we expand $\psi\left(\left|\boldsymbol{x}-\boldsymbol{z}_{r s}\right|\right)$ around $\boldsymbol{z}_{r}$, we obtain: 


$$
\sum_{\mathscr{S}_{r}}\left[\psi\left(\left|\boldsymbol{x}-\boldsymbol{z}_{r}(t)\right|\right) \sum_{\mathscr{S}_{r}} \boldsymbol{f}_{r s}(t)\right] \approx \partial_{\boldsymbol{x}} \cdot\left[n_{r}\langle\boldsymbol{M}\rangle_{p}^{r}-(1 / 2) \partial_{\boldsymbol{x}} \cdot\left(n_{r}\langle\boldsymbol{N}\rangle_{p}^{r}\right)\right]
$$

in which:

$$
\begin{gathered}
n_{r}(\boldsymbol{x}, t)\langle\boldsymbol{M}\rangle_{p}^{r}(\boldsymbol{x}, t) \equiv \sum_{\mathscr{S}_{r}}\left\{\psi\left(\left|\boldsymbol{x}-\boldsymbol{z}_{r}(t)\right|\right) r_{r} \sum_{\mathscr{S}_{r}}\left[\boldsymbol{k}_{r s}(t) \boldsymbol{f}_{r s}(t)\right]\right\} \\
n_{r}(\boldsymbol{x}, t)\langle\boldsymbol{N}\rangle_{p}^{r}(\boldsymbol{x}, t) \equiv \sum_{\mathscr{S}_{r}}\left\{\psi\left(\left|\boldsymbol{x}-\boldsymbol{z}_{r}(t)\right|\right) r_{r}^{2} \sum_{\mathscr{S}_{r}}\left[\boldsymbol{k}_{r s}(t) \boldsymbol{k}_{r s}(t) \boldsymbol{f}_{r s}(t)\right]\right\}
\end{gathered}
$$

where $\boldsymbol{k}_{r s}(t)$ denotes the versor of the vector $\boldsymbol{z}_{r s}(t)-\boldsymbol{z}_{r}(t)$.

We then define the following second-order tensor, which appears in the bracket on the right-hand side of Eq. III.27, as the collisional stress tensor of the $r$ th particle phase:

$$
\langle\boldsymbol{C}\rangle_{p}^{r} \equiv n_{r}\langle\boldsymbol{M}\rangle_{p}^{r}-(1 / 2) \partial_{\boldsymbol{x}} \cdot\left(n_{r}\langle\boldsymbol{N}\rangle_{p}^{r}\right)
$$

This term accounts for the transfer of linear momentum at collisions between particles of phase $\mathscr{S}_{r}$ over their center-to-center distance $2 r_{r}$ and is important (usually dominant) in dense suspensions.

We now consider the contact forces acting between $r$ particles of phase $\mathscr{S}_{r}$ and $s$ particles of phase $\mathscr{S}_{s}$. This term reads:

$$
\sum_{\mathscr{S}_{r}}\left[\psi\left(\left|\boldsymbol{x}-\boldsymbol{z}_{r}(t)\right|\right) \sum_{\mathscr{S}_{s}} \boldsymbol{f}_{r s}(t)\right]
$$

One may be tempted to regard it as an interaction force between phases $\mathscr{S}_{r}$ and $\mathscr{S}_{s}$. If this interpretation was correct, the action-and-reaction principle should hold and the condition below should be satisfied:

$$
\sum_{\mathscr{S}_{r}}\left[\psi\left(\left|\boldsymbol{x}-\boldsymbol{z}_{r}(t)\right|\right) \sum_{\mathscr{S}_{s}} \boldsymbol{f}_{r s}(t)\right]=-\sum_{\mathscr{S}_{s}}\left[\psi\left(\left|\boldsymbol{x}-\boldsymbol{z}_{s}(t)\right|\right) \sum_{\mathscr{S}_{r}} \boldsymbol{f}_{s r}(t)\right]
$$

This equation is not always fulfilled, inasmuch as even if $\boldsymbol{f}_{r s}=-\boldsymbol{f}_{s r}$, it is $\psi\left(\left|\boldsymbol{x}-\boldsymbol{z}_{r}\right|\right) \neq \psi\left(\left|\boldsymbol{x}-\boldsymbol{z}_{s}\right|\right)$. The condition is met only for $r_{r} \rightarrow 0$ and $r_{s} \rightarrow 0$. We conclude that the force in Eq. III.31 cannot be regarded as the interaction force between phases $\mathscr{S}_{r}$ and $\mathscr{S}_{s}$, but must include an additional part that does not satisfy the action-and-reaction principle. To obtain this force, we expand $\psi\left(\left|\boldsymbol{x}-\boldsymbol{z}_{r s}\right|\right)$ about the point $\boldsymbol{z}_{r}$, retaining only the first three terms of the expansion. Doing so allows us to write:

$$
\sum_{\mathscr{S}_{r}}\left[\psi\left(\left|\boldsymbol{x}-\boldsymbol{z}_{r}(t)\right|\right) \sum_{\mathscr{S}_{s}} \boldsymbol{f}_{r s}(t)\right] \approx n_{r}\langle\boldsymbol{f}\rangle_{p}^{r s}+\partial_{\boldsymbol{x}} \cdot\left[n_{r}\langle\boldsymbol{P}\rangle_{p}^{r s}-(1 / 2) \partial_{\boldsymbol{x}} \cdot\left(n_{r}\langle\boldsymbol{Q}\rangle_{p}^{r s}\right)\right]
$$

in which

$$
\begin{gathered}
n_{r}(\boldsymbol{x}, t)\langle\boldsymbol{f}\rangle_{p}^{r s}(\boldsymbol{x}, t) \equiv \sum_{\mathscr{S}_{r}}\left\{\psi\left(\left|\boldsymbol{x}-\boldsymbol{z}_{r s}(t)\right|\right) \sum_{\mathscr{S}_{s}} \boldsymbol{f}_{r s}(t)\right\} \\
n_{r}(\boldsymbol{x}, t)\langle\boldsymbol{P}\rangle_{p}^{r s}(\boldsymbol{x}, t) \equiv \sum_{\mathscr{S}_{r}}\left\{\psi\left(\left|\boldsymbol{x}-\boldsymbol{z}_{r}(t)\right|\right) r_{r} \sum_{\mathscr{S}_{s}}\left[\boldsymbol{k}_{r s}(t) \boldsymbol{f}_{r s}(t)\right]\right\} \\
n_{r}(\boldsymbol{x}, t)\langle\boldsymbol{Q}\rangle_{p}^{r s}(\boldsymbol{x}, t) \equiv \sum_{\mathscr{S}_{r}}\left\{\psi\left(\left|\boldsymbol{x}-\boldsymbol{z}_{r}(t)\right|\right) r_{r}^{2} \sum_{\mathscr{S}_{s}}\left[\boldsymbol{k}_{r s}(t) \boldsymbol{k}_{r s}(t) \boldsymbol{f}_{r s}(t)\right]\right\}
\end{gathered}
$$




\begin{tabular}{c}
\hline $\begin{array}{c}\text { Continuity equation - Fluid phase } \\
\partial_{t} \varepsilon+\partial_{\boldsymbol{x}} \cdot\left(\varepsilon\langle\boldsymbol{u}\rangle_{e}\right)=0\end{array}$ \\
\hline Continuity equation - Particle phase $r$ \\
$\partial_{t} \phi_{r}+\partial_{\boldsymbol{x}} \cdot\left(\phi_{r}\langle\boldsymbol{v}\rangle_{p}^{r}\right)=0$ \\
\hline Dynamical equation - Fluid phase \\
$\rho_{e}\left[\partial_{t}\left(\varepsilon\langle\boldsymbol{u}\rangle_{e}\right)+\partial_{\boldsymbol{x}} \cdot\left(\varepsilon\langle\boldsymbol{u}\rangle_{e}\langle\boldsymbol{u}\rangle_{e}\right)\right]=\partial_{\boldsymbol{x}} \cdot\langle\boldsymbol{S}\rangle_{e}-\sum_{r=1}^{\nu} n_{r}\langle\boldsymbol{f}\rangle_{p}^{r}+\varepsilon \rho_{e} \boldsymbol{g}$ \\
\hline$\rho_{r}\left[\partial_{t}\left(\phi_{r}\langle\boldsymbol{v}\rangle_{p}^{r}\right)+\partial_{\boldsymbol{x}} \cdot\left(\phi_{r}\langle\boldsymbol{v}\rangle_{p}^{r}\langle\boldsymbol{v}\rangle_{p}^{r}\right)\right]=\partial_{\boldsymbol{x}} \cdot\langle\boldsymbol{S}\rangle_{p}^{r}+n_{r}\langle\boldsymbol{f}\rangle_{p}^{r}+\sum_{s \neq r=1}^{\nu} n_{r}\langle\boldsymbol{f}\rangle_{p}^{r s}+\phi_{r} \rho_{r} \boldsymbol{g}$ \\
\hline
\end{tabular}

Table 1: Eulerian-Eulerian averaged equations of motion for a system of $\nu$ particle classes.

We define the following second-order tensor:

$$
\langle\boldsymbol{D}\rangle_{p}^{r s} \equiv n_{r}\langle\boldsymbol{P}\rangle_{p}^{r s}-(1 / 2) \partial_{\boldsymbol{x}} \cdot\left(n_{r}\langle\boldsymbol{Q}\rangle_{p}^{r s}\right)
$$

as the collisional stress tensor related to the momentum transferred at collisions between phases $\mathscr{S}_{r}$ and $\mathscr{S}_{s}$. Finally, the effective stress tensor of phase $\mathscr{S}_{r}$ can be defined as:

$$
\langle\boldsymbol{S}\rangle_{p}^{r} \equiv\langle\boldsymbol{C}\rangle_{p}^{r}+\sum_{s \neq r=1}^{\nu}\langle\boldsymbol{D}\rangle_{p}^{r s}-n_{r} \rho_{r} V_{r}\langle\hat{\boldsymbol{v}} \hat{\boldsymbol{v}}\rangle_{p}^{r}
$$

The first two terms, taken together, represent the (total) collisional stress tensor, while the last represents the kinetic stress tensor. This terms arises from the Reynolds decomposition of the convection term featuring in the averaged linear momentum balance equation.

Knowing the expressions for the components of the effective stress tensor of phase $\mathscr{S}_{r}$, we can write the averaged linear momentum balance equation for it as follows:

$$
\rho_{r} V_{r}\left[\partial_{t}\left(n_{r}\langle\boldsymbol{v}\rangle_{p}^{r}\right)+\partial_{\boldsymbol{x}} \cdot\left(n_{r}\langle\boldsymbol{v}\rangle_{p}^{r}\langle\boldsymbol{v}\rangle_{p}^{r}\right)\right]=\partial_{\boldsymbol{x}} \cdot\langle\boldsymbol{S}\rangle_{p}^{r}+n_{r}\langle\boldsymbol{f}\rangle_{p}^{r}+\sum_{s \neq r=1}^{\nu} n_{r}\langle\boldsymbol{f}\rangle_{p}^{r s}+n_{r} \rho_{r} V_{r} \boldsymbol{g}
$$

In this equation, the interaction forces between the phases (represented by the second and third terms on the right-hand side) satisfy the action-and-reaction principle. Table 1 reports, in absolute notation, the multifluid equations of motion just derived.

\section{The closure problem}

As we discussed in the previous section, the effective stress tensors of the fluid and solid phases, as well as the interaction force between the fluid and each solid phase and between each pair of solid phases, which are present in the dynamical equations of the multifluid model, are unclosed. This is because the relations which define these terms involve point variables and not volume averaged variables. So, in a macroscopic modeling context, these relations have no practical use, because they cannot be solved.

To overcome the closure problem, one has to derive expressions relating the unclosed terms to the mean variables. This can be done either using purely theoretical expressions or semi-empirical ones. The former 
are usually prohibitive to obtain, with the exception of few comparatively simple limiting cases; accordingly, Eulerian-Eulerian models heavily rely on semi-empirical relations. These are not always extremely accurate, but are able to capture the essential physics of the system and describe it with a reasonable level of accuracy (for most practical applications).

In this section, we first discuss some closures for the effective stress tensors of the fluid and solid phases. We then consider the fluid-particle interaction forces, with emphasis on the buoyancy and drag forces (which usually play a dominant role). We finally present some closures for the particle-particle interaction force. For simplicity, from now on we do not report the angular brackets that imply averaging.

\section{A Effective stress}

The effective stress tensors of all phases (fluid phase included) in general are complex to model. Researchers usually adopt the simplest functional form for them, namely the Newtonian closure, even if evidence clearly indicates that more elaborate relations are required (Jackson, 1997, 2000). Assuming that both fluid and solid phases behave as Newtonian fluids, we can write:

$$
\boldsymbol{S}_{e}=-\left[p_{e}-\left(\kappa_{e}-\frac{2}{3} \mu_{e}\right) \operatorname{tr} \boldsymbol{D}_{e}\right] \boldsymbol{I}+2 \mu_{e} \boldsymbol{D}_{e} ; \boldsymbol{S}_{r}=-\left[p_{r}-\left(\kappa_{r}-\frac{2}{3} \mu_{r}\right) \operatorname{tr} \boldsymbol{D}_{r}\right] \boldsymbol{I}+2 \mu_{r} \boldsymbol{D}_{r}
$$

in which $p_{e}, p_{r}, \kappa_{e}, \kappa_{r}, \mu_{e}$ and $\mu_{r}$ are the effective pressure, dilatational viscosity and shear viscosity of the fluid and of the $r$ th solid phase, respectively, $\boldsymbol{I}$ is the unit tensor, and $\boldsymbol{D}_{e}$ and $\boldsymbol{D}_{r}$ are the rate of deformation (or strain) tensors, defined as:

$$
\boldsymbol{D}_{e} \equiv \frac{1}{2}\left(\partial_{\boldsymbol{x}} \boldsymbol{u}_{e}+\partial_{\boldsymbol{x}} \boldsymbol{u}_{e}^{T}\right) \quad ; \quad \boldsymbol{D}_{r} \equiv \frac{1}{2}\left(\partial_{\boldsymbol{x}} \boldsymbol{v}_{r}+\partial_{\boldsymbol{x}} \boldsymbol{v}_{r}^{T}\right)
$$

The Newtonian closures have been widely employed for several fluidization regimes, being able to reproduce satisfactorily many experimental results, especially for powders far from maximum packing.

As we see from Eqs. IV.1, the closure problem now reduces to deriving suitable constitutive expressions for the pressure, dilatational viscosity and shear viscosity of each phase. For the fluid phase, one can assume that the interstitial fluid is incompressible, as we did in Section III; then, no constitutive equation is required for $p_{e}$. The dilatational viscosity is usually assumed to be zero, whilst it is assumed that $\mu_{e}=\varepsilon \bar{\mu}_{e}$, where $\bar{\mu}_{e}$ is the shear viscosity of the interstitial fluid.

For the solid phases, constitutive expressions for pressure, dilatational viscosity and shear viscosity have been derived from the kinetic theory of granular gases (Gidaspow, 1994; Brilliantov \& Poschel, 2004), which is a generalization of the mathematical theory of dense non-uniform gases (Chapman \& Cowling, 1970). As for a molecular gas, granular pressure and viscosity are functions of a granular temperature. For solid phase $\mathscr{S}_{r}$, this is defined as $\Theta_{r} \equiv(2 / 3) U_{r}$, where $U_{r}$ is the pseudointernal energy per unit mass, which is governed by the following pseudointernal energy balance equation:

$$
\rho_{r}\left[\partial_{t}\left(\phi_{r} U_{r}\right)+\partial_{\boldsymbol{x}} \cdot\left(\phi_{r} U_{r} \boldsymbol{v}_{r}\right)\right]=-\partial_{\boldsymbol{x}} \cdot \boldsymbol{q}_{r}+\boldsymbol{S}_{r} \cdot \partial_{\boldsymbol{x}} \boldsymbol{v}_{r}+G_{d, r}-S_{v, r}-S_{c, r}
$$

where $\boldsymbol{q}_{r}$ represents a pseudothermal heat flux, $G_{d, r}(\boldsymbol{x}, t)$ is a source term representing generation of particle velocity fluctuations by fluctuating fluid-particle forces, $S_{v, r}(\boldsymbol{x}, t)$ is a sink term representing the dampening of such fluctuations by the viscous resistance to particle motion, whilst $S_{c, r}(\boldsymbol{x}, t)$ is a sink term representing energy degradation caused by inelastic collisions. $\boldsymbol{q}_{r}$ is modeled using the Fourier law, writing:

$$
\boldsymbol{q}_{r}=-k_{r} \partial_{\boldsymbol{x}} \Theta_{r}
$$

where $k_{r}$ is the granular thermal conductivity of the $r$ th solid phase. Closures for this term, which are based on the kinetic theory of granular gases, can be found in the literature. For instance, Gidaspow et al. (1992) suggested the following relation: 


$$
k_{r}=\frac{150 \rho_{r} s_{r}\left(\pi \Theta_{r}\right)^{1 / 2}}{384 g_{r}\left(1+e_{r}\right)}\left[1+(6 / 5) \phi_{r} g_{r}\left(1+e_{r}\right)\right]^{2}+2 \phi_{r}^{2} \rho_{r} s_{r} g_{r}\left(1+e_{r}\right)\left(\Theta_{r} / \pi\right)^{1 / 2}
$$

where $s_{r}$ denotes the particle diameter, $e_{r}$ the coefficient of restitution for particle collisions and $g_{r}$ the radial distribution function for the $r$ th solid phase. An example of such a function, which was developed by Iddir $\&$ Arastoopour (2005), reads:

$$
g_{r}=\left[1-\left(\phi / \phi_{\max }\right)\right]^{-1}+\left(3 s_{r} / 2\right) \sum_{s=1}^{\nu}\left(\phi_{s} / s_{s}\right)
$$

in which $s_{s}$ is the particle diameter for phase $s, \phi$ is the total solid volume fraction and $\phi_{\max }$ is the maximum solid compaction (which is often taken to be equal to 0.63 ).

Constitutive equations are also required for the source and sink terms in Eq. IV.3; for briefness, we do not report them here, referring instead to the literature (for instance, Syamlal et al., 1993, Gidaspow, 1994, Fan \& Zhu, 1998 and Jackson, 2000).

The granular kinetic theory also has been adopted by several researchers to derive closures for the solid pressure. For instance, Lun et al. (1984) suggested the following expression:

$$
p_{r}=\left[1+2 \sum_{s=1}^{\nu}\left(s_{r s} / s_{r}\right)^{3} \phi_{s} g_{r s}\left(1+e_{r s}\right)\right] \phi_{r} \rho_{r} \Theta_{r}
$$

in which $e_{r s}$ is the coefficient of restitution for collisions between particles belonging to phases $\mathscr{S}_{r}$ and $\mathscr{S}_{s}$, and where the following definitions are given:

$$
s_{r s} \equiv\left(s_{r}+s_{s}\right) / 2 \quad ; \quad g_{r s} \equiv\left(s_{r} g_{s}+s_{s} g_{r}\right) /\left(s_{r}+s_{s}\right)
$$

For the solid-phase dilatational and shear viscosities, there are many constitutive equations available. Those suggested by Gidaspow (1994), which are often employed, are:

$$
\begin{gathered}
\kappa_{r}=(4 / 3) \phi_{r}^{2} \rho_{r} s_{r} g_{r}\left(1+e_{r}\right)\left(\Theta_{r} / \pi\right)^{1 / 2} \\
\mu_{r}=\frac{10 \rho_{r} s_{r}\left(\pi \Theta_{r}\right)^{1 / 2}}{96 g_{r}\left(1+e_{r}\right)}\left[1+(4 / 5) \phi_{r} g_{r}\left(1+e_{r}\right)\right]^{2}+(4 / 5) \phi_{r}^{2} \rho_{r} s_{r} g_{r}\left(1+e_{r}\right)\left(\Theta_{r} / \pi\right)^{1 / 2}
\end{gathered}
$$

Using the kinetic theory framework to derive constitutive equations for fluidized suspensions makes physical sense, because granular gases resemble molecular gases in many ways. However, the similarity breaks down for dense suspensions, and therefore, in the dense limit, closures based on the kinetic theory are expected to fail. In (granular) kinetic theory, particles are assumed to be smooth and spherical, and collisions to be binary and instantaneous. These assumptions are valid in dilute systems, in which the transfer of linear momentum is translational and collisional. In dense systems, particles undergo enduring contacts (which usually involve several neighbors) and momentum transfer is primarily frictional. In this "frictional flow regime", granular kinetic theory is clearly inadequate.

To model the solid stress tensor in the frictional flow regime, researchers normally resort to empirical or phenomenological models based on the theory of soil mechanics. One assumes that the solid phase acts as a solid body below a critical value of the magnitude of the stress tensor; above this threshold value (referred to as yield stress), the solid phase is assumed to behave as a fluid, which therefore flows under shear. Usually, in these flow conditions, the frictional stress tensor is expressed as follows:

$$
\boldsymbol{S}_{r}^{\star}=-p_{r}^{\star} \boldsymbol{I}+2 \mu_{r}^{\star} \boldsymbol{D}_{r}
$$

where the star indicates that the quantity refers to the frictional flow regime. So, two quantities are required to close the problem: frictional pressure and frictional viscosity. Srivastava \& Sundaresan (2003) suggested the so-called "Princeton model" for the frictional pressure: 


$$
p_{r}^{\star}=\phi_{r} p^{\star} \quad, \quad p^{\star}= \begin{cases}0 & \text { for } \phi_{r} \leq \phi_{\mathrm{fr}} \\ A\left(\phi_{r}-\phi_{\mathrm{fr}}\right)^{B}\left(\phi_{\max }-\phi_{r}\right)^{C} & \text { for } \phi_{\mathrm{fr}}<\phi_{r} \leq \phi_{\max } \\ 10^{D}\left(\phi_{r}-\phi_{\mathrm{fr}}\right)^{E} & \text { for } \phi_{r}>\phi_{\max }\end{cases}
$$

where $\phi_{\mathrm{fr}}$ denotes the critical frictional solid packing (the solid volume fraction threshold value at which the powder enters the frictional flow regime). In the relation for $p^{\star}$ given on the second line, which was proposed by Johnson \& Jackson (1987), $A, B$ and $C$ are constants with typical values of $0.05,2$ and -5 , respectively. In the relation for $p^{\star}$ given on the last line, which was suggested by Syamlal et al. (1993), the coefficients $D$ and $E$ are very large, with typical values of 25 and 10, respectively. Many other constitutive equations for the frictional pressure have been proposed; for these, the reader is referred to the literature.

The expression used for the frictional shear viscosity in the Princeton model is based on soil mechanics (Tardos, 1997; Jackson, 2000) are reads:

$$
\mu_{r}^{\star}=\frac{p_{r}^{\star} \sin \vartheta_{r}}{2 \sqrt{I_{2}\left(\boldsymbol{D}_{r}\right)+\Theta_{r} / s_{r}^{2}}}\left(\sum_{s=1}^{\nu} \phi_{s}\right)^{-1} \quad, \quad I_{2}\left(\boldsymbol{D}_{r}\right) \equiv \frac{1}{2}\left[\left(\operatorname{tr} \boldsymbol{D}_{r}\right)^{2}-\operatorname{tr} \boldsymbol{D}_{r}^{2}\right]
$$

in which $\vartheta_{r}$ is the angle of internal friction of the $r$ th solid phase, while $I_{2}\left(\boldsymbol{D}_{r}\right)$ is the second invariant of the rate of deformation tensor of the $r$ th solid phase. If only one solid phase is present and the contribution of the term involving the granular temperature is negligible, this equation simplifies to that originally proposed by Schaeffer (1987). For other constitutive equations, we refer to the literature.

Often, in the frictional flow regime, the kinetic and collisional stress contributions, modeled via granular kinetic theory, are summed to the frictional stress to deliver the total stress, although the first two are usually negligible compared to the third.

\section{B Fluid-particle interaction force}

The fluid-particle interaction force can be divided into five main contributors:

- Buoyancy force, whose definition for fluid-particle systems is not unique and will be discussed.

- Local fluid acceleration force, which is parallel to the mean acceleration of the fluid and is present only when a specific definition for the buoyancy force is adopted.

- Drag force, which acts in the direction of the fluid-particle slip velocity (i.e., the fluid velocity relative to an observer moving with the same local mean velocity as the particles).

- Virtual mass force, which is parallel to the relative acceleration between the phases.

- Lift force, which is normal to the slip velocity.

Among these contributors, usually the buoyancy and drag forces are dominant.

\section{B.I Buoyancy force}

There are three different definitions for the buoyancy force:

- The first regards the force to be equal to the weight of the fluid displaced by the particles. This is in line with the Archimedes principle; therefore, we call this classical definition. The force per unit volume of suspension then takes the form:

$$
n_{r} \boldsymbol{f}_{B, r}^{\star} \equiv-\phi_{r} \rho_{e} \boldsymbol{g}
$$


- The second definition relates the force to the effective stress tensor of the fluid phase; per unit volume of suspension, it is:

$$
n_{r} \boldsymbol{f}_{B, r}^{\bullet} \equiv \phi_{r} \partial_{\boldsymbol{x}} \cdot \boldsymbol{S}_{e}
$$

- The third definition, often encountered in the literature, retains solely the isotropic part of the effective fluid stress tensor; the closure therefore takes the form:

$$
n_{r} \boldsymbol{f}_{B, r}^{\circ} \equiv-\phi_{r} \partial_{\boldsymbol{x}} p_{e}
$$

These expressions, of course, lead to different values of the buoyancy force; this is perfectly fine, because we are free to define a force as we wish. What is important is that the total fluid-particle interaction force $n_{r} \boldsymbol{f}_{r}$, which has objective physical meaning, is calculated correctly. Consequently, modelers who adopt different definitions of the buoyancy force also need to adopt different expressions for the complementary force that makes up the overall fluid-particle interaction force. Therefore, for instance, if one opts to employ Eq. IV.16, the contribution of the deviatoric part of $\boldsymbol{S}_{e}$ in Eq. IV.15 has to be included in the complementary force; this contribution, therefore, is still present, but is regarded as part of the complementary force.

\section{B.II Local fluid acceleration force}

If the classical definition of buoyancy force is employed, the complementary force to the (total) fluid-particle interaction force must include a term known as local fluid acceleration force (this term is absent otherwise). Per unit volume of suspension, this force is given by:

$$
n_{r} \boldsymbol{f}_{A, r}^{\star} \equiv \phi_{r} \rho_{e} D_{t}^{e} \boldsymbol{u}_{e} \quad, \quad D_{t}^{e} \boldsymbol{u}_{e} \equiv \partial_{t} \boldsymbol{u}_{e}+\boldsymbol{u}_{e} \cdot \partial_{\boldsymbol{x}} \boldsymbol{u}_{e}
$$

$D_{t}^{e} \boldsymbol{u}_{e}$ is the material (or substantial) derivative relative to a Lagrangian observer that moves with the locally averaged velocity of the fluid.

\section{B.III Drag force}

The drag force is defined as the fluid-particle interaction force component that is parallel to the slip velocity between the phases. In general, it reads:

$$
n_{r} \boldsymbol{f}_{D, r} \equiv \beta_{r}\left(\boldsymbol{u}_{e}-\boldsymbol{v}_{r}\right)
$$

where $\beta_{r}$ is the drag coefficient for the $r$ th solid phase. We now present some of the expressions suggested in the literature for $\beta_{r}$, written so that the associated drag force is consistent with the classical definition of the buoyancy force. To obtain the value of $\beta_{r}$ consistent with the buoyancy force definition IV.15, one needs to multiply the expressions for $\beta_{r}$ given here by $\varepsilon$ (Mazzei \& Lettieri, 2007).

Ergun \& Orning (1949) advanced the empirical correlation below, initially developed for pressure drops through packed beds and later used for uniform fluid beds:

$$
\beta_{r}=150 \frac{\mu_{e} \phi_{r}(1-\varepsilon)}{\left(\varepsilon s_{r}\right)^{2}}+1.75 \frac{\rho_{e} \phi_{r}\left|\boldsymbol{u}_{e}-\boldsymbol{v}_{r}\right|}{\varepsilon s_{r}}
$$

This equation was developed, and has been extensively verified, for fixed beds (in which the void fraction is low, close to 0.40); however, Gidaspow (1994) suggests using this closure for void fraction values up to 0.80 . For larger values, he suggests using the expression of Wen \& Yu (1966), which is one of the most popular in the literature; this closure reads:

$$
\beta_{r}=\frac{3}{4} C_{D}\left(\operatorname{Re}_{r}\right) \frac{\rho_{e} \phi_{r}\left|\boldsymbol{u}_{e}-\boldsymbol{v}_{r}\right|}{s_{r}} \varepsilon^{-2.70}
$$


where:

$$
\operatorname{Re}_{r} \equiv \frac{\rho_{e} \varepsilon\left|\boldsymbol{u}_{e}-\boldsymbol{v}_{r}\right| s_{r}}{\mu_{e}} \quad ; \quad C_{D}\left(\operatorname{Re}_{r}\right)= \begin{cases}\left(24 / \operatorname{Re}_{r}\right)\left(1+0.15 \mathrm{Re}_{r}^{0.687}\right) & \text { for } \operatorname{Re}_{r}<1000 \\ 0.44 & \text { for } \operatorname{Re}_{r} \geq 1000\end{cases}
$$

Here, $\operatorname{Re}_{r}$ denotes the Reynolds number associated with the particles of solid phase $\mathscr{S}_{r}$. The expression for the coefficient $C_{D}$ was advanced by Schiller \& Naumann (1935).

In Eq. IV.20, the exponent $\alpha$ in the voidage function $\varepsilon^{-\alpha}$ is constant. Di Felice (1994) suggested that $\alpha$ should be a function of $\mathrm{Re}_{r}$. The empirical expression that he proposed is:

$$
\alpha\left(\operatorname{Re}_{r}\right)=2.70-0.65 \exp \left[-(1 / 2)\left(1.50-\log _{10} \operatorname{Re}_{r}\right)^{2}\right]
$$

As we see, for very small and very large values of the Reynolds number, $\alpha$ approaches 2.70 . However, in the intermediate region, the deviation from 2.70 is significant. For instance, the exponent reaches a minimum value of 2.05 for $\operatorname{Re}_{r} \approx 32$.

The Richardson \& Zaki (1954) equation is very accurate at describing the expansion of uniform fluidized beds of non-cohesive particles. The closures reported above, however, are inconsistent with it. To overcome this limitation, Mazzei \& Lettieri (2007) derived an expression that is consistent with it over the entire range of fluid-dynamic regimes and for any value of the void fraction. This closure, which for vary small and very large values of $\mathrm{Re}_{r}$ reduces to the Wen \& Yu (1966) equation, can be expressed as per Eq. IV.20, where the exponent in the voidage function is given by:

$$
\alpha\left(\varepsilon, \operatorname{Re}_{r}\right)=-(1 / \ln \varepsilon) \ln \left\{\frac{\left[0.63+4.80\left(\operatorname{Re}_{r} / \varepsilon^{\gamma}\right)^{-1 / 2}\right]^{2}}{\left(0.63+4.80 \operatorname{Re}_{r}^{-1 / 2}\right)^{2}} \varepsilon^{2(1-\gamma)}\right\}
$$

in which:

$$
\gamma\left(\varepsilon, \operatorname{Re}_{r}\right)=\frac{4.80+2.40 \cdot 0.175\left(\operatorname{Re}_{r} / \varepsilon^{\gamma}\right)^{3 / 4}}{1+0.175\left(\operatorname{Re}_{r} / \varepsilon^{\gamma}\right)^{3 / 4}}
$$

For a detailed discussion about this closure and how its results compare with the other constitutive equations reported above, we refer to Mazzei \& Lettieri (2007).

\section{B.IV Virtual mass force}

The acceleration of a body immersed in a fluid leads to the acceleration of the latter. This results into a force, called virtual mass force, that has the following form:

$$
n_{r} \boldsymbol{f}_{V, r} \equiv \phi_{r} \rho_{e} C_{V}\left(\phi_{r}\right)\left(D_{t}^{e} \boldsymbol{u}_{e}-D_{t}^{r} \boldsymbol{v}_{r}\right)
$$

where $D_{t}^{e}(\cdot)$ and $D_{t}^{r}(\cdot)$ are the material derivatives associated with the fluid and $r$ th solid phase, respectively. The virtual mass coefficient $C_{V}\left(\phi_{r}\right)$ for a single sphere immersed in an infinite fluid is equal to $1 / 2$ (Maxey $\&$ Riley, 1983). This value is also used for very dilute mixtures of spherical particles (for instance, see Zhang $\&$ Prosperetti, 1994, who obtained this value for inviscid flows). $C_{V}\left(\phi_{r}\right)$, however, in general depends on the shape of the particles and on the volume fraction of the solid phase; in particular, the coefficient is expected to be an increasing function of the latter. For moderate values of the particle volume fraction, Zuber (1964) suggested the following linear relation:

$$
C_{V}\left(\phi_{r}\right)=\left(1+3 \phi_{r}\right) / 2
$$

The virtual mass force plays an important role when the density of the fluid is comparable with or larger than that of the disperse phase; in gas-fluidized beds, therefore, it is negligible. 


\section{B.V Lift force}

When an object moves in a fluid which is in shearing flow, it experiences a force transverse to the direction of relative motion. This lift force is equal to:

$$
n_{r} \boldsymbol{f}_{L, r} \equiv \phi_{r} \rho_{e} C_{L}\left(\phi_{r}\right)\left(\boldsymbol{u}_{e}-\boldsymbol{v}_{r}\right) \times\left(\partial_{\boldsymbol{x}} \times \boldsymbol{u}_{e}\right)
$$

The lift coefficient $C_{L}\left(\phi_{r}\right)$ also depends on the particle shape and on the volume fraction of the solid phase. For very dilute mixtures of spherical particles, $C_{L}\left(\phi_{r}\right)$ is also taken to be $1 / 2$. This is because the sum of the virtual mass and lift forces has to satisfy the principle of material objectivity (Drew \& Passman, 1998); each force taken individually does not, but their sum does if $C_{L}\left(\phi_{r}\right)=C_{V}\left(\phi_{r}\right)$.

\section{B.VI Other forces}

Other contributions to the fluid-particle force are present; for a comprehensive overview, the reader can refer to Drew \& Passman (1998). The expression for them are usually for a single particle in an infinite fluid. The Faxen force, the elastic force (Foscolo \& Gibilaro, 1984, 1987; Mazzei et al., 2006) and a history-dependent force analogous to that of Basset for single particles (Basset, 1888) are examples of such forces.

\section{Particle-particle interaction force}

If several monodisperse particle classes are present in a fluidized suspension, each of them exchanges linear momentum with all the others through particle collisions (and friction, if the suspension is dense). This force is usually modeled as a particle-particle "drag force", assuming that it is parallel to the slip velocity between each pair of particle classes. One thus write:

$$
n_{r} \boldsymbol{f}_{r s} \equiv \zeta_{r s}\left(\boldsymbol{v}_{s}-\boldsymbol{v}_{r}\right)
$$

where $n_{r} \boldsymbol{f}_{r s}$ is the force exerted by phase $s$ on phase $r$ per unit volume of suspension (see Table 1) and $\zeta_{r s}$ is the particle-particle drag coefficient for the two particle classes involved. Gidaspow et al. (1985) advanced the following expression for $\zeta_{r s}$ :

$$
\zeta_{r s}=C_{r s}\left(1+e_{r s}\right)\left[\frac{\phi_{r} \phi_{s} \rho_{r} \rho_{s}\left(s_{r}+s_{s}\right)^{2}}{\rho_{r} s_{r}^{3}+\rho_{s} s_{s}^{3}}\right]\left|\boldsymbol{v}_{s}-\boldsymbol{v}_{r}\right|
$$

which is based on granular kinetic theory calculations (and so cannot describe accurately the particle-particle interactions in dense suspensions). In this equation, it is:

$$
C_{r s} \equiv \frac{3 \Phi_{r s}^{1 / 3}+\left(\phi_{r}+\phi_{s}\right)^{1 / 3}}{4\left[\Phi_{r s}^{1 / 3}-\left(\phi_{r}+\phi_{s}\right)^{1 / 3}\right]}
$$

where:

$$
\begin{gathered}
\Phi_{r s} \equiv\left(1-s_{r s}\right)\left[\Phi_{r}+\left(1-\Phi_{r}\right) \Phi_{s}\right]\left(1-X_{r s}\right)+\Phi_{r} \quad \text { for } \quad X_{r s} \geq \frac{\Phi_{r}}{\Phi_{r}+\left(1-\Phi_{r}\right) \Phi_{s}} \\
\Phi_{r s} \equiv\left[\left(\Phi_{r}-\Phi_{s}\right)+\left(1-s_{r s}\right)\left(1-\Phi_{r}\right) \Phi_{s}\right]\left[\Phi_{r}+\left(1-\Phi_{r}\right) \Phi_{s}\right] \frac{X_{r s}}{\Phi_{r}}+\Phi_{s} \text { otherwise }
\end{gathered}
$$

In the relations above, $\Phi_{r}$ and $\Phi_{s}$ are the particle volume fractions at maximum packing for phases $r$ and $s$, respectively; moreover:

$$
X_{r s} \equiv \frac{\phi_{r}}{\phi_{r}+\phi_{s}} \quad ; \quad s_{r s} \equiv\left(\frac{s_{s}}{s_{r}}\right)^{1 / 2} \quad \text { if } s_{r} \geq s_{s} \text { and } s_{r s} \equiv\left(\frac{s_{r}}{s_{s}}\right)^{1 / 2} \text { otherwise }
$$


Another popular closure is that of Syamlal (1987), which reads:

$$
\zeta_{r s}=\frac{3}{4}\left(1+e_{r s}\right)\left(1+\frac{\pi}{4} F_{r s}\right)\left[\frac{\phi_{r} \phi_{s} \rho_{r} \rho_{s} g_{r s}\left(s_{r}+s_{s}\right)^{2}}{\rho_{r} s_{r}^{3}+\rho_{s} s_{s}^{3}}\right]\left|\boldsymbol{v}_{s}-\boldsymbol{v}_{r}\right|
$$

where $F_{r s}$ is a coefficient of friction for phases $r$ and $s$. In the equation above, the constitutive equation used for the radial distribution function is that of Lebowitz (1964), which is given by:

$$
g_{r}=(1 / \varepsilon)\left[1+\left(3 s_{r} / 2 \varepsilon\right) \sum_{s=1}^{\nu}\left(\phi_{s} / s_{s}\right)\right] \quad ; \quad g_{r s} \equiv\left(s_{r} g_{s}+s_{s} g_{r}\right) /\left(s_{r}+s_{s}\right)
$$

Being based on the kinetic theory of granular gases, Eqs. IV.29 and IV.33 are not valid for dense suspensions, and in particular in the frictional flow regime. Nonetheless, sometimes they are used also in these conditions, when the system approaches the maximum packing limit. Doing so is incorrect. Notice that, in a system that is nearly packed, particles of different size may not segregate; Eqs. IV.29 and IV.33, however, do not prevent segregation from occurring, because the drag coefficient $\zeta_{r s}$ does not diverge in the dense limit. To overcome the problem, as suggested by Gera et al. (2004), one can add, to the expressions for $\zeta_{\text {rs }}$ given above, the term $\Psi p^{\star}$, where $p^{\star}$ is given by Eq. IV.12 and $\Psi$ is a coefficient whose value should be obtained empirically. This term ensures that, when a powder approaches maximum packing, the particle-particle drag increases enough to make the solid phases $r$ and $s$ move at the same velocity, as if they were one phase. This prevents particle segregation. Gera et al. (2004) set $\Psi$ equal to 0.30 , but this value has no general validity.

\section{Population balance modeling}

The Eulerian equations of motion presented in Section III are based on the assumption that all the particles in the suspension are identical. All the particles, in particular, must have the same size, and this cannot change in time. This is a significant limitation of this modeling approach, because in real systems particles can grow, shrink, aggregate and break, and new particles may form. This means that the particles do not have the same size, and their particle size distribution (PSD) changes in space and time, its evolution reflecting the physical and chemical processes taking place in the system. Capturing this evolution, consequently, is necessary for a more realistic description of multiphase systems. A powerful modeling approach that offers this capability is the population balance modeling approach.

Let us consider a population of particles in which the state of each particle is characterized by its velocity in real space $\boldsymbol{v}$ and its size $s$ (additional variables can be considered, but for simplicity we restrict the analysis to these two). The state of the entire particle population is described by the so-called number density function (NDF), which we denote as $f(\boldsymbol{v}, s, \boldsymbol{x}, t)$. This function is defined so that $f(\boldsymbol{v}, s, \boldsymbol{x}, t) d \boldsymbol{v} d s d \boldsymbol{x}$ represents the expected (in a statistical sense) number of particles located at time $t$ in the volume $d \boldsymbol{x}$ about the point $\boldsymbol{x}$ with velocity in the range $d \boldsymbol{v}$ about the velocity $\boldsymbol{v}$ with size in the range $d s$ about the size $s$. So, knowing the NDF is equivalent to knowing (in a statistical sense) the state of the entire particle population in any spatial point of physical space and at any time.

The population balance equation governs the evolution of the NDF. There are two main ways of deriving it. In the first, one begins from the microscopic description of the particulate system and obtains the equation via statistical averaging; details about this rigorous, but quite complex, approach can be found in (Marchisio \& Fox, 2013). The other derivation method is heuristic and consists in writing a continuity statement for the NDF in the phase space of one particle (this space, in our case, has six dimensions: three in real space, three in velocity space and one in size space). Here, we follow this second method.

Let us define the six-dimensional vector $\boldsymbol{r} \equiv(\boldsymbol{x}, \boldsymbol{v}, s)$. The first three components of $\boldsymbol{r}$ are those of $\boldsymbol{x}$, the three immediately after are those of $\boldsymbol{v}$ and the final one coincides with $s$. We find it convenient to regard the vector $\boldsymbol{r}$ as the position vector of one particle in the abstract six-dimensional space in which such a particle moves. Let us now consider three arbitrary fixed control volumes, one in real space, one in velocity space and one in size space; we denote them as $\Lambda_{x}, \Lambda_{v}$ and $\Lambda_{s}$, respectively; the corresponding control volume in phase 
space is $\Lambda_{r} \equiv \Lambda_{x} \cup \Lambda_{v} \cup \Lambda_{s}$. We now aim to write a number balance equation for the particle population over this six-dimensional control volume. The number of particles that accumulate in it per unit time is:

$$
A C C=\partial_{t} \int_{\Lambda_{r}} f d \boldsymbol{r}=\int_{\Lambda_{r}} \partial_{t} f d \boldsymbol{r}
$$

The net number of particles entering $\Lambda_{r}$ per unit time is:

$$
I N-O U T=-\int_{\partial \Lambda_{r}} \boldsymbol{n}_{r} \cdot \dot{\boldsymbol{r}} f d \boldsymbol{s}_{r}=-\int_{\Lambda_{r}} \partial_{\boldsymbol{r}} \cdot f \dot{\boldsymbol{r}} d \boldsymbol{r}
$$

in which $\dot{\boldsymbol{r}}$ and $\partial_{\boldsymbol{r}}$ are the particle velocity and the nabla operator in phase space, respectively, $\boldsymbol{n}_{r}$ is the unit vector normal to the hypersurface bounding $\Lambda_{r}$ directed outwards and $d \boldsymbol{s}_{r}$ is an infinitesimal surface element on $\partial \Lambda_{r}$. We have used the Gauss theorem to turn the surface integral into a volume integral.

If $\mathbb{G}_{r}$ denotes the net number of particles generated per unit volume of phase space (owing, for instance, to particle collisions, aggregation, breakage or nucleation), we can write:

$$
G E N=\int_{\Lambda_{r}} \mathbb{G}_{r} d \boldsymbol{r}
$$

The processes modeled through this generation term are regarded as instantaneous (no process, of course, is instantaneous, but these processes have characteristic times that are so shorter than those characterizing the evolution of the NDF that we can regard them as instantaneous).

The difference between the accumulation (Eq. V.1) and convection (Eq. V.2) terms, must be equal to the generation term. Thus, we can write:

$$
\int_{\Lambda_{r}}\left(\partial_{t} f+\partial_{\boldsymbol{r}} \cdot f \dot{\boldsymbol{r}}-\mathbb{G}_{r}\right) d \boldsymbol{r}=0
$$

Because the integration volume $\Lambda_{r}$ is arbitrary and the integrand is (assumed to be) continuous, we conclude that the integrand must be equal to zero; accordingly, it is:

$$
\partial_{t} f=-\partial_{\boldsymbol{r}} \cdot f \dot{\boldsymbol{r}}+\mathbb{G}_{r}
$$

This is the population balance equation (PBE). If we let $\dot{v}$ and $\dot{s}$ denote the particle velocity in velocity space and size space (the first coincides with the particle acceleration in real space and the second with the particle growth in real space), respectively, we can write the equation above as:

$$
\partial_{t} f=-\partial_{x} \cdot f \boldsymbol{v}-\partial_{\boldsymbol{v}} \cdot f \dot{\boldsymbol{v}}-\partial_{s}(f \dot{s})+\mathbb{G}_{r}
$$

Solving the PBE allows determining the NDF evolution. The equation, nonetheless, can be solved only if it is closed; by closed we mean that all the terms present in the equation, such as the generation term, are known functions of the NDF. Deriving these closures is quite complex, especially for the generation term; for details about the closure problem, we refer to Ramkrishna (2000) and Marchisio \& Fox (2013).

When the PBE is closed, it can be solved, but doing so is extremely difficult and demanding, because in general it is a nonlinear, integral, partial differential, functional equation in several dimensions. Therefore, one does not usually attempt to solve it, using the equation to extract solely the information about the system behavior which is of interest in the application at hand. Often one is interested only in few integral properties of the NDF; called moments, these might be important because they relate to the product quality or because they are easy to measure and monitor. The idea behind the so-called method of moments is deriving evolution equations for the moments of interest by integrating out the coordinates $v$ and $s$ from the PBE. Doing so is not complex; nevertheless, usually the evolution equations that one obtains are unclosed (even if the PBE is closed), because for any given set of moments that a modeler wishes to track, the set of evolution equations for these moments involves moments external to the set considered. Because of this problem, the method of moments, in its original formulation, has been seldom used. 
To overcome the closure problem, one may assume a functional form for the NDF and use the evolution equations for the moments to determine the parameters that are left unspecified in the expression adopted for the density function. Let us clarify this concept. In the method of moments, one renounces to solve the PBE, solving instead a number of evolution equations for the moments of interest of the NDF. These equations permit calculating the moments of interest in every point of the system at every time, but they do not permit determining the NDF, because an infinite number of density functions can yield the same set of moments (as long as the set is finite). This leaves the NDF largely undetermined, and so we are free (to a certain extent) to choose the functional form of the density function arbitrarily and then use the moment evolution equations to determine the details which we have left unspecified. The various moment methods differ in the choice of the functional form assumed for the NDF.

The quadrature methods of moments are examples of this approach; they overcome the closure problem by assuming that the NDF has the following functional expression:

$$
f(\boldsymbol{v}, s, \boldsymbol{x}, t)=\sum_{r=1}^{\nu} n_{r}(\boldsymbol{x}, t) \boldsymbol{\delta}\left[\boldsymbol{v}-\boldsymbol{v}_{r}(\boldsymbol{x}, t)\right] \delta\left[s-s_{r}(\boldsymbol{x}, t)\right]
$$

This is a quadrature formula, in which $\nu$ is the number of nodes, $\boldsymbol{v}_{r}(\boldsymbol{x}, t)$ and $s_{r}(\boldsymbol{x}, t)$ are the $r$ th quadrature nodes and $n_{r}(\boldsymbol{x}, t)$ is the $r$ th quadrature weight. This formula represents the particle population by means of $\nu$ solid phases, the $r$ th having number density $n_{r}(\boldsymbol{x}, t)$ and being made up of particles with velocity $\boldsymbol{v}_{r}(\boldsymbol{x}, t)$ and size $s_{r}(\boldsymbol{x}, t)$. The difference between this representation and that used in Section III is that here the size of each particle class is not fixed, but varies in time and real space. The $3 \nu$ parameters $n_{r}(\boldsymbol{x}, t), \boldsymbol{v}_{r}(\boldsymbol{x}, t)$ and $s_{r}(\boldsymbol{x}, t)$ are determined using the evolution equations of $3 \nu$ moments. For details about how this is done, we refer the interested reader to Marchisio \& Fox (2013).

\section{Conclusions}

We presented several strategies for modeling fluidized beds. Some of these describe in detail the dynamics of of the system, but are too expensive computationally to be of any use in practical applications - in particular those of industrial relevance. The Eulerian-Eulerian modeling approach, conversely, is based on equations that can be solved at reasonable computational cost and that can deliver observables of direct interest to most end-users. We derived the volume-averaged balance equations for mass and linear momentum for fluidized mixtures made up of $\nu$ particle classes. We then addressed the closure problem, presenting some constitutive equations that modelers adopt to express the effective stress of each phase and the interaction forces between the phases. We concluded the paper by introducing the population balance modeling approach, which allows describing systems where the particles are continuously distributed over the size (or any other property that characterizes the particle state), and in which the size is free to vary owing to continuous and discontinuous processes, such as chemical reaction, growth, aggregation and breakage.

\section{Bibliography}

Ahmadi G, Ma D (1990) A thermodynamical formulation for dispersed multiphase turbulent flows-1: Basic theory. Int. J. Multiph. Flow. 16:323-340 .

Allen MP, Tildesley DJ (1990) Computer Simulations of Liquids. Oxford Science Publications, New York.

Anderson TB, Jackson R (1967) A fluid mechanical description of fluidized beds. Equations of motion. Ind. Eng. Chem. Fundam. 6:527-539.

Balescu R (1975) Equilibrium and Nonequilibrium Statistical Mechanics. Wiley, New York.

Basset AB (1888) A treatise on Hydrodynamics. Deighton Bell, London. 
Bird RB, Stewart WE, Lightfoot EN (1960) Transport Phenomena. Wiley, New York.

Börner M, Bück A, Tsotsas E (2017) DEM-CFD investigation of particle residence time distribution in top-spray fluidised bed granulation. Chem. Eng. Sci. 161:187-197.

Brilliantov NV, Poschel T (2004) Kinetic Theory of Granular Gases. Oxford University Press, Oxford.

Buyevich YA (1971) Statistical hydrodynamics of disperse systems. Part 1. Physical background and general equations. J. Fluid Mech. 49:489-507.

Chapman S, Cowling TG (1970) The Mathematical Theory of Non-Uniform Gases. Cambridge University Press, Cambridge.

Cundall PA, Strack OD (1979) A discrete numerical model for granular assemblies. Géotechnique 29:47-65.

Deen NG, Peters EAJF, Padding JT, Kuipers JAM (2014) Review of direct numerical simulation of fluidparticle mass, momentum and heat transfer in dense gas-solid flows. Chem. Eng. Sci. 116:710-724.

Di Felice R (1994) The voidage function for fluid-particle interaction systems. Int. J. Multiphase Flow 20:153-159.

Di Renzo A, Cello F, Di Maio FP (2011) Simulation of the layer inversion phenomenon in binary liquidfluidized beds by DEM-CFD with a drag law for polydisperse systems. Chem. Eng. Sci. 66:2945-2958.

Drew DA (1971) Averaged field equations for two-phase media. Stud. Appl. Math. 50:133-166.

Drew DA, Lahey RT (1993) Analytical modelling of multiphase flow. In Particulate Two-Phase Flow. Butterworth-Heinemann, Boston.

Drew DA, Passman SL (1998) Theory of Multicomponent Fluids. Applied Mathematical Sciences. Springer, New York.

Drew DA, Segel LA (1971) Averaged equations for two-phase flows. Stud. Appl. Math. 50:205-231.

Drew DA (1983) Mathematical modelling of two-phase flow. Annu. Rev. Fluid Mech. 15:261-291.

Enwald H, Peirano E, Almstedt AE (1996) Eulerian two-phase flow theory applied to fluidization. Int. J. Multiphase Flow 22:21-66.

Ergun S, Orning AA (1949) Fluid flow through randomly packed columns and fluidized beds. Ind. Eng. Chem. 41:1179-1184.

Fan LS, Zhu C (1998) Principles of Gas-Solid Flows. Cambridge University Press, Cambridge.

Feng Z-G, Alatawi Eid S, Roig A, Sarikaya C (2016) A Resolved Eulerian-Lagrangian Simulation of Fluidization of 1204 Heated Spheres in a Bed With Heat Transfer. J. Fluid. Eng.-T. ASME. 138:041305.

Foscolo P U, Gibilaro L G (1984) A fully predictive criterion for the transition between particulate and aggregate fluidization. Chem. Eng. Sci. 39:1667-1675.

Foscolo P U, Gibilaro L G (1987) Fluid dynamic stability of fluidized suspensions: The particle bed model. Chem. Eng. Sci. 42:1489-1500.

Fries L, Antonyuk S, Heinrich S, Dopfer D, Palzer S (2013) Collision dynamics in fluidised bed granulators: A DEM-CFD study. Chem. Eng. Sci. 86:108-123.

Gera D, Syamlal M, O’Brien TJ (2004) Hydrodynamics of particle segregation in fluidized beds. Int. J. Multiphase Flow. 30:419-428.

Gidaspow D (1994) Multiphase Flow and Fluidization. Academic Press, London. 
Gidaspow D, Ettehadieh B (1983) Fluidization in two-dimensional beds with a jet. Part II: hydrodynamic modeling. Ind. Eng. Chem. Fundam. 22:193-201.

Gidaspow D, Bezburuah R, Ding J (1992) Hydrodynamics of circulating fluidized beds. Kinetic theory apparoach. In proc. of the 7th International Conference on Fluidization. Brisbane, Australia.

Gidaspow D, Syamlal M, Seo YC (1985) Hydrodynamics of fluidization of single and binary particles: supercomputer modeling. In proc. of the 5th International Conference on Fluidization. Elsinore, Denmark.

Gidaspow D, Syamlal M, Seo YC (1986) Hydrodynamics of fluidization: supercomputer generated vs. experimental bubbles. J. Powder \& Bulk Solids Tech. 10:19-23.

Hassani M.A, Zarghami R, Norouzi H.R, Mostoufi N (2013) Numerical investigation of effect of electrostatic forces on the hydrodynamics of gas-solid fluidized beds. Powder Technol. 246:16-25.

He Y, Wang T, Deen N, van Sint Annaland M, Kuipers JAM, Wen D (2012) Discrete particle modeling of granular temperature distribution in a bubbling fluidized bed. Particuology 10:428-437.

Hinch EJ (1977) An averaged equation approach to particle interactions in a fluid suspension. J. Fluid Mech. 83:695-720.

Hoomans BPB, Kuipers JAM, Briels WJ, van Swaaij WPM (1996) Discrete particle simulation of a twodimensional gas-fluidized bed: a hard sphere approach. Chem. Eng. Sci. 51:99-118.

Hwang G.J., Shen H.H., (1989) Modeling the solid phase stress in a fluid-solid mixture. Int. J. Multiph. Flow 15:257-268.

Iddir H, Arastoopour H (2005) Modeling of multitype particle flow using the kinetic theory approach. AIChE J. 51:1620-1632.

Ishii M, Mishima K (1984) Two-fluid model and hydrodynamic constitutive relations. Nucl. Eng. and Des.82:107-126.

Ishii M, Takashi H (2010) Thermo-Fluid Dynamic Theory of Two-Phase Flow. Springer.

Ismail T. M, Abd El-Salam M, Monteiro E, Rouboa A (2016) Eulerian-Eulerian CFD model on fluidized bed gasifier using coffee husks as fuel. Appl. Therm. Eng. 106:1391-1402.

Jackson R (1997) Locally averaged equations of motion for a mixture of identical spherical particles and a Newtonian fluid. Chem. Eng. Sci. 52:2457-2469.

Jackson R (1998) Erratum. Chem. Eng. Sci. 53:1955.

Jackson R (2000) The Dynamics of Fluidized Particles. Cambridge Monographs on Mechanics. Cambridge University Press, Cambridge.

Jenkins JT, Savage SB (1983) A theory for the rapid flow of identical, smooth, nearly elastic, spherical particles. J. Fluid Mech. 130:187-202.

Johnson, P.C., Jackson, R., 1987. Frictional-Collisional Constitutive Relations for Granular Materials, with Application to Plane Shearing. J. Fluid Mech. 176:67-93.

Kafui KD, Thornton C, Adams MJ (2002) Discrete particle-continuum fluid modelling of gas-solid fluidised beds. Chem. Eng. Sci. 57:2395-2410.

Lebowitz JL (1964) Exact solution of generalised Percus-Yevick equation for a mixture of hard spheres. Phys. Rev. 133:895-899.

Leva M (1959). Fluidization. McGraw-Hill, New York. 
Lu H, Wang S, Zhao Y, Yang L, Gidaspow D, Ding J (2005) Prediction of particle motion in a twodimensional bubbling fluidized bed using hard-sphere model. Chem. Eng. Sci. 60:3217-3231.

Lun CKK, Savage SB, Jeffrey DJ, Chepurniy N (1984) Kinetic theories for granular flow: Inelastic particles in Couette flow and slightly inelastic particles in a general flow field. J. Fluid Mech. 140:223-256.

Marchioro M, Tanksley M, Prosperetti A (1999) Mixture pressure and stress in disperse two-phase flow. Int. J. Multiphas. Flow 25:1395-1429.

Marchisio DL, Fox RO (2013) Computational Models for Polydisperse Particulate and Multiphase Systems. Cambridge University Press, Cambridge.

Massoudi Farid M, Jeong J. H, Kim K. H, Lee J, Kim D, Hwang J (2017) Numerical investigation of particle transport hydrodynamics and coal combustion in an industrial-scale circulating fluidized bed combustor: Effects of coal feeder positions and coal feeding rates. Fuel 192:187-200.

Maxey MR, Riley JJ (1983) Equation of motion for a small rigid sphere in a nonuniform flow. Phys. Fluids 26:883-889.

Mazzei L, Lettieri P (2007) A drag force closure for uniformly-dispersed fluidized suspensions. Chem. Eng. Sci. 62:6129-6142.

Mazzei L, Lettieri P, Elson T, Colman D (2006) A revised monodimensional particle bed model for fluidized beds. Chem. Eng. Sci. 61:1958-1972.

Nigmatulin RI (1979) Spatial averaging in the mechanics of heterogeneous and dispersed systems. Int. J. Multiphase Flow. 5:353-385.

Ouyang J, Li J (1999) Particle-motion-resolved discrete model for simulating gas-solid fluidization. Chem. Eng. Sci. 54:2077-2083.

Owoyemi O, Mazzei L, Lettieri P (2007) CFD modeling of binary-fluidized suspensions and investigation of role of particle-particle drag on mixing and segregation. AIChE J. 53:1924-1940.

Pan TW, Joseph DD, Bai R, Glowinski R, Sarin V (2002) Fluidization of 1204 spheres: simulation and experiments. J. Fluid Mech. 451:169-191.

Pandit JK, Wang XS, Rhodes MJ (2005) Study of Geldart's Group A behaviour using the discrete element method simulation. Powder Technol. 160:7-14.

Pritchett JW, Blake TR, Garg SK (1978) A numerical model of gas fluidized beds. AIChE Symp. Ser. 176:134-148.

Ramkrishna D (2000) Population Balances. Academic Press, London.

Richardson JF, Zaki WN (1954) Sedimentation and fluidization: Part I. Trans. Inst. Chem. Eng. 32:35-53.

Sangani A.S, Didwania A.K(1993) Dispersed-phase stress tensor in flows of bubbly liquids at large Reynolds numbers. J. Fluid Mech. 248:27-54.

Sankaranarayanan K, Shan X, Kevrekidis IG, Sundaresan S (2002) Analysis of drag and virtual mass forces in bubbly suspensions using an implicit formulation of the lattice Boltzmann method. J. Fluid Mech. 452:61-96.

Schaeffer DG (1987) Instability in the evolution equations describing incompressible granular flow. J. Diff. Eq. 66:19-50.

Schiller L, Naumann Z (1935) A drag coefficient correlation. Z. Ver. Deutsch. Ing. 77:318-320. 
Schneiderbauer S, Pirker S, Puttinger S, Aguayo P, Touloupidis V, Joaristi A.M (2016) A LagrangianEulerian hybrid model for the simulation of poly-disperse fluidized beds: Application to industrial-scale olefin polymerization. Powder Technol. In press.

Schneiderbauer S, Puttinger S, Pirker S (2015) Numerical Study of a Bi-disperse Gas-solid Fluidized Bed Using an Eulerian and Lagrangian Hybrid Model. Procedia Eng. 102: 1539-1545.

Srivastava, A., Sundaresan, S., 2003. Analysis of a frictional-kinetic model for gas-particle flow. Powder Technol. 129:72-85.

Syamlal M (1987) The particle-particle drag term in a multiparticle model of fluidization. National Technical Information Service. DOE/MC/21353-2373, NTIS/DE87006500.

Syamlal M, Rogers WA, O’Brien TJ (1993) MFIX Documentation and Theory Guide. DOE/METC94/1004, NTIS/DE94000087. Electronically available from: http://www.mfix.org.

Tardos G I (1997) A fluid mechanistic approach to slow, frictional flow of powders. Powder Technol. 92:61-74.

Tsuji Y, Kawaguchi T, Tanaka T (1993) Discrete particle simulation of two-dimensional fluidized bed. Powder Technol. 77:79-87.

van der Hoef MA, Beetstra R, Kuipers JAM (2005) Lattice Boltzmann simulations of low Reynolds number flow past mono- and bidisperse arrays of spheres: results for the permeability and drag force. J. Fluid. Mech. 528:233-254.

Wang J, van der Hoef MA, Kuipers JAM (2013) Particle granular temperature of Geldart A, A/B and B particles in dense gas-fluidized beds. Chem. Eng. Sci. 97:264-271.

Wen CY, Yu YH (1966) Mechanics of Fluidization. Chem. Eng. Prog. Symp. Ser. 62:100-111.

Whitaker S (1969) Advances in the theory of fluid motion in porous media. Ind. Eng. Chem. 61:14-28.

Xu BH, Yu AB (1997) Numerical simulation of the gas-solid flow in a fluidized bed by combining discrete and particle method with computational fluid dynamics. Chem. Eng. Sci. 52:2785-2809.

Yang Y, Zi C, Huang Z, Wang J, Lungu M, Liao Z, Yang Y, Su H (2017) CFD-DEM investigation of particle elutriation with electrostatic effects in gas-solid fluidized beds. Powder Technol. 308:422-433.

Ye M, van der Hoef MA, Kuipers JAM (2005) The effects of particle and gas properties on the fluidization of Geldart A particles. Chem. Eng. Sci. 60:4567-4580.

Zhang DZ, Prosperetti A (1994) Averaged equations for inviscid disperse two-phase flow. J. Fluid. Mech. 267:185-219.

Zhao Y, Li G, Luo Z, Zhang B, Dong L, Liang C, Duan C (2017) Industrial application of a modularized Dry-Coal-Beneficiation technique based on a novel air dense medium fluidized bed . Int. J. Coal Prep. Util. 37: 44-57.

Zhou L, Zhang L, Bai L, Shi W, Wang C, Agarwal R (2017) Experimental study and transient CFD/DEM simulation in a fluidized bed based on different drag models. RSC Adv. 7:12764-12774.

Zhu HP, Zhou ZY, Yang RY, Yu AB (2008) Discrete particle simulation of particulate systems: a review of major applications and findings. Chem. Eng. Sci. 63:5728-5770.

Zuber N (1964) On dispersed two-phase flow in the laminar flow regime. Chem. Eng. Sci. 19:897-903. 